\title{
RANK-ONE PERTURBATIONS OF NORMAL OPERATORS AND HYPONORMALITY
}

\author{
Il Bong Jung AND Eun Young LeE
}

Abstract. Let $T=N+u \otimes v$ be a rank-one perturbation of a normal operator $N$ acting on a separable, infinite dimensional, complex Hilbert space $\mathscr{H}$. It is proved that the hyponormality of $T$ is equivalent to the normality of $T$. Some characterizations of hyponormality[normality] of $T$ are obtained.

Mathematics subject classification (2010): Primary 47B20, 47A63; Secondary 47A55.

Keywords and phrases: Normal operator, hyponormal operator, rank-one perturbation, commutator.

\section{REFERENCES}

[1] W. Donoghue, On the perturbation of spectra, Comm. Pure Appl. Math. 18 (1965), 559-579.

[2] E. EXNER, I. B. JUNG, E. Y. LEE, AND M. R. LEE, Gaps of operators via rank-one perturbations, J. Math. Anal. Appl. 376 (2011), 576-587.

[3] C. Foias, I. B. Jung, E. Ko, AND C. PEARCY, On rank-one perturbations of normal operators, J. Funct. Anal. 253 (2007), 628-646.

[4] C. FoIAs, I. B. JUnG, E. Ko, AND C. PEARCY, On rank-one perturbations of normal operators, II, Indiana Univ. Math. J. 57 (2008), 2745-2760.

[5] C. Foias, I. B. Jung, E. Ko, And C. PEARCy, Spectral decomposability of rank-one perturbations of normal operators, J. Math. Anal. Appl. 375 (2011), 602-609.

[6] E. IONASCU, Rank-one perturbations of diagonal operators, Integr. Equat. Oper. Th. 39 (2001), 421440.

[7] T. FURUTA, Invitation to Linear Operators, Taylor \& Francis Inc., London/New York, 2001.

[8] S. JitOMIRSKAYA AND B. Simon, Operators with singular continuous spectrum, III; almost periodic Schrödinger operators, Comm. Math. Phys. 165 (1994), 201-205.

[9] E. Ko And J. E. LEE, On rank-one perturbations of unilateral shift, Commun. Kor. Math. Soc. 26 (2011), 79-88.

[10] C. PEARCy, Some Recent Developments in Operator Theory, C.B.M.S. Regional Conference Series in Mathematics, No. 36, Amer. Math. Soc., Providence, Rhode Island, 1978.

[11] R. Del Rio, N. Makarov And B. Simon, Operators with singular continuous spectrum, II; rank one operators, Comm. Math. Phys. 165 (1994), 59-67.

[12] J. G. StAmPFl, One-dimensional perturbations of operators, Pacific J. Math., 115 (1984), $481-491$.

[13] J. Weidmann, Linear Operators in Hilbert Spaces, Springer-Verlag, Berlin, Heidelberg, New York, 1980. 